(C) 2015 IEEE. Personal use of this material is permitted. Permission from IEEE must be obtained for all other uses, in any current or future media, including reprinting/republishing this material for advertising or promotional purposes, creating new collective works, for resale or redistribution to servers or lists, or reuse of any copyrighted component of this work in other works. 


\title{
Single Channel Wireless EEG Device for Real-Time Fatigue Level Detection
}

\author{
Li-Wei Ko ${ }^{1,2^{*}}$, Wei-Kai Lai ${ }^{1,2}$, Wei-Gang Liang ${ }^{1,2}$, Chun-Hsiang Chuang ${ }^{1}$, Shao-Wei Lu ${ }^{1}$, Yi-Chen Lu ${ }^{1,2}$, Tien-Yang \\ Hsiung $^{3}$, Hsu-Hsuan $\mathrm{Wu}^{3 *}$, Chin-Teng Lin ${ }^{1 *}$ \\ ${ }^{1}$ Brain Research Center and I-RiCE Center, National Chiao-Tung University, Hsinchu, Taiwan \\ ${ }^{2}$ Institute of Bioinformatics and Systems Biology, National Chiao-Tung University, Hsinchu, Taiwan \\ ${ }^{3}$ AmTRAN Technology Co.,Ltd. New Taipei City, Taiwan \\ *Corresponding Email to: $\underline{\text { ctlin@mail.nctu.edu.tw, 1wko@mail.nctu.edu.tw, sam.wu@amtran.com.tw }}$
}

\begin{abstract}
Driver fatigue problem is one of the important factors of traffic accidents. Recent years, many research had investigated that using EEG signals can effectively detect driver's drowsiness level. However, real-time monitoring system is required to apply these fatigue level detection techniques in the practical application, especially in the real-road driving. Therefore, it required less channels, portable and wireless, realtime monitoring and processing techniques for developing the real-time monitoring system. In this study, we develop a single channel wireless EEG device which can real-time detect driver's fatigue level on the mobile device such as smart phone or tablet. The developed device is investigated to obtain a better and precise understanding of brain activities of mental fatigue under driving, which is of great benefit for devolvement of detection of driving fatigue system. This system consists of a Bluetoothenabled one channel EEG, a regression model, and smartphone, which was a platform recording and transforming the raw EEG data to useful driving status. In the experiment, this was a sustained-attention driving task to implement in a virtual-reality (VR) driving simulator. To training model and develop the system, we were performed for 15 subjects to study Electroencephalography (EEG) brain dynamics by using a mobile and wireless EEG device. Based on the outstanding training results, the leave-one-subject-out cross validation test obtained $90 \%$ fatigue detection accuracy. These results indicate that the combination of a smartphone and wireless EEG device constitutes an effective and easy wearable solution for detecting and preventing driver fatigue in real driving environments.
\end{abstract}

Keywords - driver drowsiness detection, Brain computer interface, wearable devices

\section{INTRODUCTION}

Fatigue driving is one of the most possible factors for traffic accidents. Some studies show almost $25 \%$ to $30 \%$ traffic accidents are related to fatigue driving [1], so how to reduce this kind of accident is one important issue for scientist. In recent years, researchers have developed some methods to enhance driving safety, like parking sensors and camera [2, 3]. But the methods mention before are from external aspect. If we can detect driver's fatigue state, it will be possible to prevent drivers from driving in their fatigue state in advance. Some physiological features, like brain wave, eye-blinking frequency, heart rate and blood pressure, are verified to have high correlation with drivers' fatigue level. By using specific devices to recording these physiological signals, we can monitor drivers' fatigue state and reduce traffic accidents caused by fatigue driving [4-8].

In the past, there are many fatigue detecting systems based on Electroencephalography (EEG). Traditional EEG is used to find the relation between brain dynamic changes in fatigue cognition function [9] and the EEG sensors need to be filled with conductive gel before recording data which is not convenient for drivers. However, some researchers start to use wireless EEG to design real-time fatigue detection BCI system which can be implemented in smart devices [10, 11, 12]. Because the number of iPhone and Android smart phone users has an unprecedented increase in 2007 and 2008, it will be familiar for people to use this system by smart phones.

Studies in this field usually use multiple channels in frontal or occipital region to establish the system and sometimes get poor accuracy. The goal of this study is to develop a signal channel EEG-based real-time fatigue detection system implemented in the smart phone. The work is divided into two main parts: 1) Recording drivers' fatigue brain wave by one channel EEG cap, U-Wake, and set up a fatigue detection regression model. 2) Establish one reliable and accurate realtime fatigue prediction system implemented in smart phones.

\section{MATERIAL AND METHODS}

\section{A. Subjects}

The fifteen volunteer subjects (aged 22 to 28 years) participated in the VR-based highway-driving experiment. All subjects had no history of gastrointestinal, cardiovascular, neurological or psychological disorders, were healthy and had no prior experience with EEG-based experiment. All experiments were conducted in the evening after dinner. They practiced the driving task for 5 min to become acquainted with the experiment procedures. 


\section{B. Experimental Task}

A sustained-attention driving task implemented the eventrelated lane-departure paradigm on the driving simulator [13]. The VR scenes simulated driving at a constant speed (100 $\mathrm{km} / \mathrm{hr}$.) on a highway with the car randomly drifting away from the center of the cruising lane to simulate driving on nonideal road surfaces or with poor alignment [14]. The used paradigm was to try to induce subjects' drowsiness and obtain their drowsy patterns, including EEG signals and behaviors. During a $1 \mathrm{hr}$. experiment task, randomly makes the car drift away from the original cruising lane and toward the left or the right side. Participants were instructed to compensate for the trajectory error as soon as possible while they detect the deviation event. The duration time in response to the deviation event, denoted as the reaction time (RT), was evaluated as an indicator to determine subject's fatigue level.

\section{EEG and Behavior Data Recording}

To record EEG signal, the server with Bluetooth module wirelessly received EEG signals from U-Wake device (Fig. 1), which had one channel in frontal area. Data was collected with a sampling rate of $512 \mathrm{~Hz}$. In the behavior data, the server also used RS-232 compatible serial port to record the 8-bit digital resolution including the start experiment (242), deviation onset (251/252 for left and right side of the deviation), and response onset (253). They were synchronized with the EEG data for further event-related analysis.

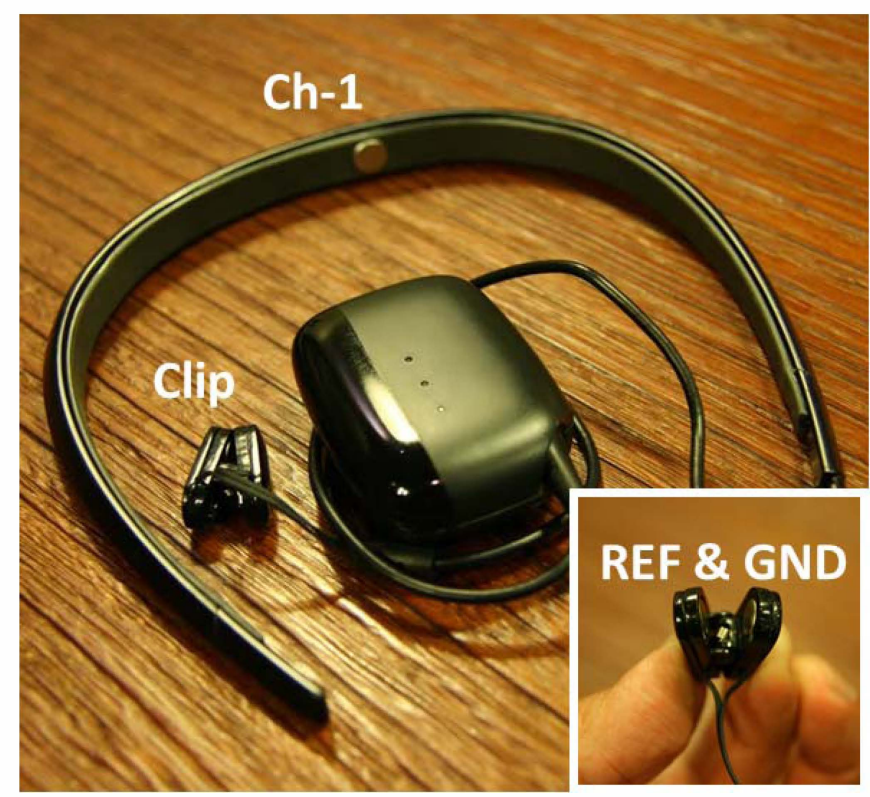

Fig. 1. Mobile and wireless EEG device (called U-Wake) with one EEG channel and one clip, which including a ground channel (GND) and a reference channel (REF).

\section{Data Processing}

Fig. 2 shows the flowchart of EEG signal processing including data acquisition, preprocessing, and prediction model. The data analysis process is listed below:

1. Band-pass filter $0.5-30 \mathrm{~Hz}$ was applied on the raw data;

2. To reduce the data size, the EEG data were downsampled to $256 \mathrm{~Hz}$;

3. Epoch extraction of 2-second data prior to the deviation onset;

4. In the first $5 \mathrm{~min}$ of experiment, epochs were used to record the baseline for normalization;

5. The fast Fourier transform (FFT) was accomplished by using 128 points Hamming window with 64 points overlap in frequency range of $1-30 \mathrm{~Hz}$;

Then, the prediction model for training is listed below:

1. The independent variables were the power spectrum array (1-30 Hz) and dependent variables were the RTs;

2. Finally, the linear regression was implemented in prediction module.

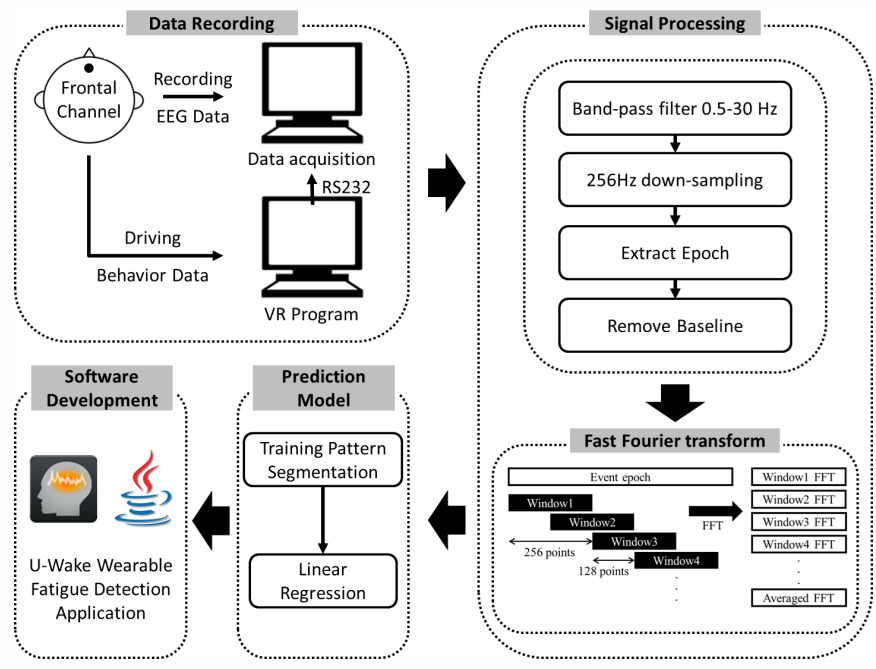

Fig. 2. Flowchart of EEG signal processing and prediction regression model

\section{E. Driver Fatigue Prediction System}

Fig. 3 shows the proposed driver fatigue prediction system flowchart, which consists of a wireless EEG based device and smartphone (i.e. android or iOS system). In this study, the wireless EEG real-time detects brain signal and smartphone is 
TABLE I

5\% SHORTEST AND LONGEST RESPONSE TIME OF ALL SUBJECTS

\begin{tabular}{|c|c|c|c|c|c|c|c|c|c|c|c|c|c|c|c|}
\hline & $\begin{array}{c}\text { Subject } \\
1\end{array}$ & $\begin{array}{c}\text { Subject } \\
2\end{array}$ & $\begin{array}{c}\text { Subject } \\
\mathbf{3}\end{array}$ & $\begin{array}{c}\text { Subject } \\
\mathbf{4}\end{array}$ & $\begin{array}{c}\text { Subject } \\
5\end{array}$ & $\begin{array}{c}\text { Subject } \\
6\end{array}$ & $\begin{array}{c}\text { Subject } \\
7 \\
\end{array}$ & $\begin{array}{c}\text { Subject } \\
8 \\
\end{array}$ & $\begin{array}{c}\text { Subject } \\
9\end{array}$ & $\begin{array}{c}\text { Subject } \\
10\end{array}$ & $\begin{array}{c}\text { Subject } \\
11\end{array}$ & $\begin{array}{c}\text { Subject } \\
12\end{array}$ & $\begin{array}{c}\text { Subject } \\
13\end{array}$ & $\begin{array}{c}\text { Subject } \\
14\end{array}$ & $\begin{array}{c}\text { Subject } \\
15\end{array}$ \\
\hline $\begin{array}{l}\text { 5\% shortest } \\
\text { RT(ms) }\end{array}$ & 477.3 & 420.9 & 423.2 & 501.4 & 482.3 & 542.6 & 565.8 & 534.6 & 565.6 & 617.5 & 463.1 & 527.6 & 693.6 & 476.9 & 783.1 \\
\hline $\begin{array}{l}\text { 5\% longest } \\
\text { RT(ms) }\end{array}$ & 696.6 & 642.2 & 663.4 & 806.2 & 818.8 & 923.3 & 947.2 & 972.9 & 1015.6 & 1117.9 & 983.6 & 1188.0 & 1423.4 & 1402.1 & 1773.4 \\
\hline $\begin{array}{l}\text { RT difference } \\
\text { (LRT-SRT) }\end{array}$ & 219.2 & 221.3 & 240.2 & 304.8 & 336.5 & 380.7 & 381.4 & 438.3 & 450.0 & 500.4 & 520.5 & 660.4 & 729.8 & 925.2 & 990.2 \\
\hline
\end{tabular}

a Bluetooth master which scans and connects to EEG device. In the data processing, the fast Fourier transformation (FFT) was used to extract EEG features. To obtain an accurate estimation, we proposed a regression model for on-line fatigue state detection. Finally, the smartphone provided a neurofeedback tool for driver self-tracking. Moreover, if the driver fatigue level exceeded a particular threshold, a warning can be activated.

\section{EXPERIMENTAL RESULTS}

In this study, all subject's reaction time (RT) to the event in the experiment was collected. The 5\% shortest and fastest RT of each subject was extracted, and sorted RT difference of shortest and fastest RT was caculated as the behavioral result, showed in the Table I. The EEG data of shortest and fastest RT was compared to discuss the different brain dynamics in two behavioral conditions. Beacause there was individual difference between 15 subjects, we intended to focus on the RT difference to observe if there was any changes of subjects during the experiment. We suggested that the RT difference would be bigger, if the subject's state became more fatigue.
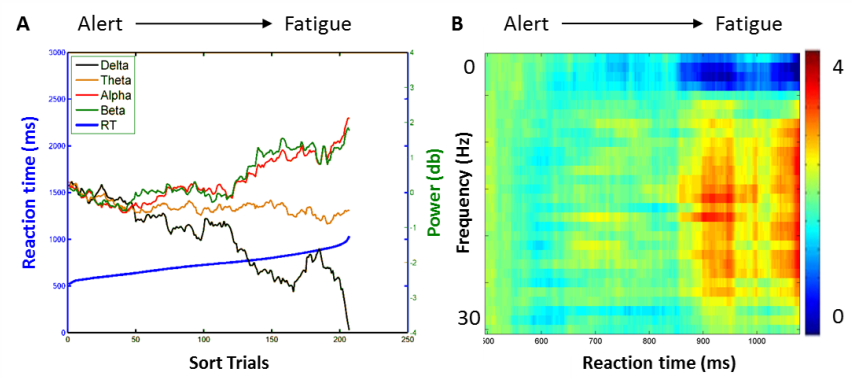

Fig. 4. (A) Power spectra of delta, theta, alpha and beta band sorted by sorted RT (B) The power spectral image for one frontal channel.

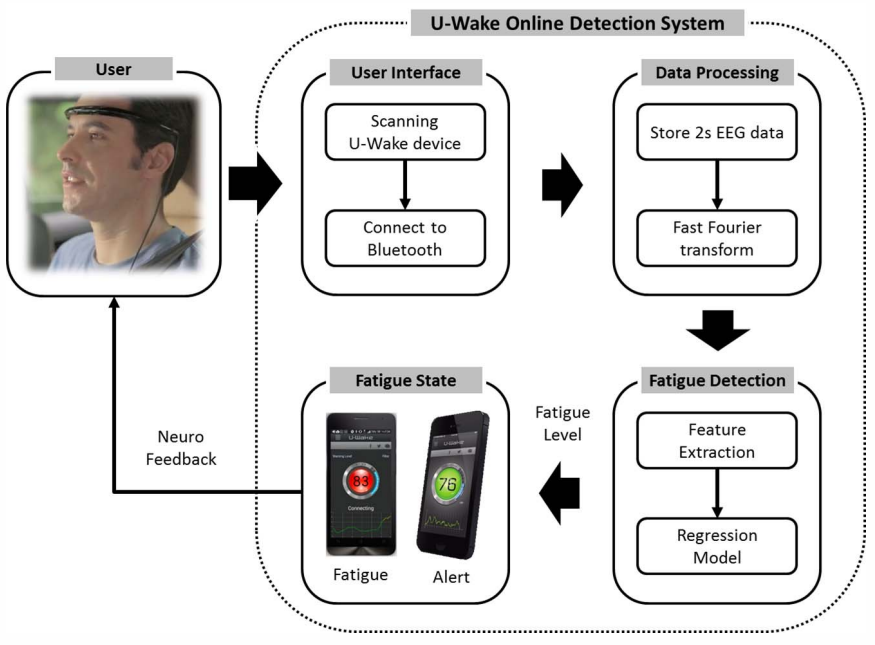

Fig. 3. Systematic diagram of a driver's vigilance prediction system

After the behavioral result, subject's frequency power was calculated to know their brain dynamic changes. In this study, frequency power was divdied into four bands, delta $(1 \sim 3 \mathrm{~Hz})$, theta $(4 \sim 8 \mathrm{~Hz})$, alpha $(9 \sim 12 \mathrm{~Hz})$ and beta $(13 \sim 30 \mathrm{~Hz})$. We plotted power of each band and sorted RT (Fig. 4A), frequency power from $0 \sim 30 \mathrm{~Hz}$ (Fig. 4B) of subjects in experiment. Power of subjects in figure $4 \mathrm{~A}$ and $4 \mathrm{~B}$ was sorted by RT. Figure $4 \mathrm{~A}$ showed the comparison of four power spectra obtained from one frontal channel. An increasing trend of frequency power could be observed in figure $4 \mathrm{~A}$ and $4 \mathrm{~B}$ when $\mathrm{RT}$ increased. The increasing phenomenon of alpha band was more obvious than other bands. Figure 4A showed RT changes with time and we could see increasing RT during this experiment which represented subject became more fatigue. In figure 5, the correlation coefficient of power and RT was shown and sorted by 15 subject's RT difference. Alpha and beta band had higher correlation coefficient than other two bands, and the high coefficent trend was more consistent in larger RT difference region. 
TABLE II

Results of The Prediction Using LEAVE-ONE-SUbJeCt-out Cross VALIDATION

\begin{tabular}{|c|c|c|}
\hline \multirow{2}{*}{ Subject } & \multicolumn{2}{|c|}{ Leave-one-subject-out validation } \\
\hline & RMSE (ms) & Accuracy \\
\hline 1 & 55.728 & 0.997 \\
\hline 2 & 118.47 & 0.996 \\
\hline 3 & 58.521 & 0.997 \\
\hline 4 & 93.659 & 0.991 \\
\hline 5 & 166.667 & 0.972 \\
\hline 6 & 217.819 & 0.953 \\
\hline 7 & 343.401 & 0.882 \\
\hline 8 & 106.6 & 0.989 \\
\hline 9 & 221.313 & 0.963 \\
\hline 10 & 149.071 & 0.978 \\
\hline 11 & 430.517 & 0.815 \\
\hline 12 & 236.801 & 0.958 \\
\hline 13 & 367.607 & 0.865 \\
\hline 14 & 329.502 & 0.891 \\
\hline 15 & 403.955 & 0.837 \\
\hline Mean & 219.975 & 0.939 \\
\hline Std. & 123.158 & 0.061 \\
\hline
\end{tabular}

Finally, the accuracy and root mean square error (RMSE) of prediction regression model was shown in table II. We divided RT into five levels, 0-1000, 1000-1500, 1500-2000, 2000-2500 and 3000- ms. If the predicted RT was in the same RT level with observed RT, the prediction would be counted as one correct prediction. The prediction model was established by leave-one-subject-out cross validation. The averaged accuracy was $93.9 \%$ in this study. Except few subjects, most subjects' accuracy were more than $90 \%$, and the highest accuracy could reach $99.7 \%$. It showed that the regression model in this study was a promising method to predict user's fatigue level.

\section{GRAPHICAL USER INTERFACE (GUI) DESIGN}

With our results, the fatigue prediction system was a feasible way to predict people's fatigue level. In order to help user can operate it easily without any professional support, we attempted to make the smartphone GUI (Fig. 6) simple to use. This GUI application included the displaying, predicating,
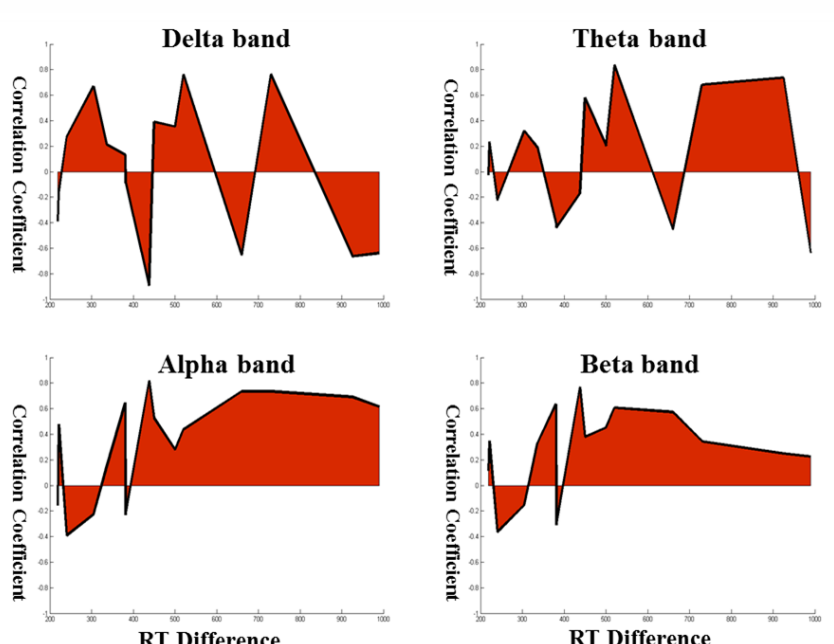

Fig. 5. The correlation coefficient of RT and power in four frequency band. The coefficient is sorted by RT difference, and it is increased in larger $\mathrm{RT}$ difference region in alpha and beta band.

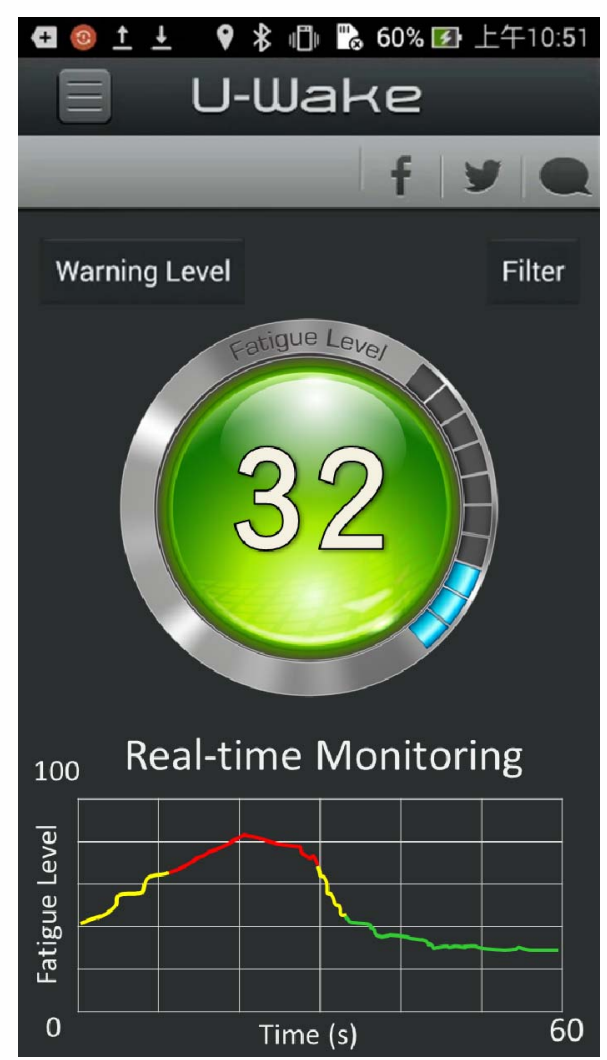

Fig. 6. The demonstration of the proposed driver fatigue prediction system. 
recording, and a neuro-feedback tool. Raw data were also recording by GUI program installed in a smartphone and saved to the Secure Digital Memory Card (SD card). Specifically, the green circle and yellow circle indicate alertness and early warning. When the driver was in the full warning state, the red circle was appearing, and additionally a long-lasting sound and vibration warning. The smartphone also will collect 2-s EEG signals to estimate the fatigue level, and show the result by the line chart at bottom of the screen. In the line chart, green line represents alertness state and red line mean fatigue state.

\section{CONCLUSIONS}

In this study, one wireless EEG-based device, U-Wake, and virtual-reality driving experiment were used to find subject's fatigue brain dynamic feature. According to the correlation between frequency power obtained by one frontal channel and $\mathrm{RT}$, the linear regression model was proposed to establish a fatigue prediction system realized by GUI in the smartphone. This system was wireless, wearable and only used one EEG channel which maintained good signal quality simultaneously, so it was suitable application to be used in real driving situations. Results also showed that it's a promising system to predict user's fatigue level, based on the high accuracy and low RMSE.

\section{ACKNOWLEDGMENTS}

This work was supported in part by the UST-UCSD International Center of Excellence in Advanced Bioengineering sponsored by the Taiwan National Science Council I-RiCE Program under Grant Number: MOST-1032911-I-009-101, in part by the Aiming for the Top University Plan of National Chiao Tung University, the Ministry of Education, Taiwan, and in part by the National Science Council, Taiwan, under Contract MOST 102-2420-H-009 003-MY3. Research was also sponsored in part by the Army Research Laboratory and was accomplished under Cooperative Agreement Number W911NF-10-2-0022.

\section{REFERENCES}

[1] T. R. S. for the Prevention of Accidents, Driver fatigue and road accidents: A literature review and position paper, Birmingham, U.K., 2001.

[2] Eriksson, Martin, and N. P. Papanikotopoulos. "Eye-tracking for detection of driver fatigue." Intelligent Transportation System, 1997. ITSC'97., IEEE Conference on. IEEE, 1997.

[3] SHI, Shu-ming, et al. "Driver mouth monitoring method based on machine vision [J]." Journal of Jilin University of Technology (Natural Science Edition) 2 (2004): 014

[4] Lin, Chin-Teng, et al. "EEG-based drowsiness estimation for safety driving using independent component analysis." Circuits and Systems I: Regular Papers, IEEE Transactions on 52.12 (2005): 2726-2738.

[5] Swarnkar, Vinayak, Udantha Abeyratne, and Craig Hukins. "Objective measure of sleepiness and sleep latency via bispectrum analysis of EEG." Medical \& biological engineering \& computing 48.12 (2010): 1203-1213.
[6] C. Iber, A. I. Sonia, L. Andrew, J. Chesson and S. F. Quan. "The AASM manual for the scoring of sleep and associated events." American academy of sleep medicine, Westchester, USA., 2007.

[7] Khushaba, Rami N., et al. "Driver drowsiness classification using fuzzy wavelet-packet-based feature-extraction algorithm." Biomedical Engineering, IEEE Transactions on 58.1 (2011): 121-131.

[8] Roy, Raphaëlle N., Sylvie Charbonnier, and Stéphane Bonnet. "Eye blink characterization from frontal EEG electrodes using source separation and pattern recognition algorithms." Biomedical Signal Processing and Control 14 (2014): 256-264.

[9] Lal, Saroj KL, et al. "Development of an algorithm for an EEG-based driver fatigue countermeasure." Journal of Safety Research 34.3 (2003): 321-328.

[10] Wang, Y. T., et al. "Cell-phone based drowsiness monitoring and management system." 2012 IEEE on Biomedical Circuits and Systems Conference (BioCAS). 2012.

[11] Bowman, Darrell, et al. Development and Assessment of a Driver Drowsiness Monitoring System. No. FMCSA-RRR-12-008. 2012

[12] Chuang, Chun-Hsiang, et al. "Independent Component Ensemble of EEG for Brain-Computer Interface." Neural Systems and Rehabilitation Engineering, IEEE Transactions on 22.2 (2014): 230-23

[13] Huang, R. S., et al. "Tonic and phasic brain dynamics during responses to simulated driving challenges." NeuroImage 47 (2009): S103.

[14] Lin, Chin-Teng, et al. "Development of wireless brain computer interface with embedded multitask scheduling and its application on real-time driver's drowsiness detection and warning." Biomedical Engineering, IEEE Transactions on 55.5 (2008): 1582-1591. 